\title{
S1PR5 is pivotal for the homeostasis of patrolling monocytes
}

\author{
Emilie Debien*1,2,3, Katia Mayol ${ }^{* 1,2,3}$, Vincent Biajoux ${ }^{4,5}$, \\ Cécile Daussy ${ }^{1,2,3}$, Mercedes Gomez De Aguero ${ }^{1,2,3}$, Morgan Taillardet ${ }^{1,2,3}$, \\ Nicolas Dagany ${ }^{1,2,3}$, Lilia Brinza ${ }^{1,2,3}$, Thomas Henry ${ }^{1,2,3}$, \\ Bertrand Dubois $^{1,2,3}$, Dominique Kaiserlian ${ }^{1,2,3}$, Jacqueline Marvel $^{1,2,3}$, \\ Karl Balabanian ${ }^{4,5}$ and Thierry Walzer ${ }^{1,2,3}$ \\ ${ }^{1}$ Université de Lyon, France \\ 2 INSERM U1111, Lyon, France \\ ${ }^{3}$ Université Lyon1, UMS3444 / US8, Lyon, France \\ ${ }^{4}$ Univ. Paris-Sud, Laboratoire "Cytokines, Chimiokines et Immunopathologie", UMR_S996, \\ Clamart, France \\ ${ }^{5}$ INSERM, Laboratory of Excellence in Research on Medication and Innovative Therapeutics \\ (LERMIT), Clamart, France
}

Patrolling Ly6C- monocytes are blood-circulating cells that play a role in inflammation and in the defense against pathogens. Here, we show that similar to natural killer (NK) cells, patrolling monocytes express high levels of S1PR5, a G-coupled receptor for sphingosine-1 phosphate. We found that S1 pr5 $5^{-/-}$mice lack peripheral Ly6C ${ }^{-}$monocytes but have a normal number of these cells in the bone marrow (BM). Various lines of evidence exclude a direct contribution of S1PR5 in the survival of Ly6C ${ }^{-}$monocytes at the periphery. Rather, our data support a role for S1PR5 in the egress of Ly6C- monocytes from the BM. In particular, we observed a reduced frequency of patrolling monocytes in BM sinusoids of S1PR5 KO mice. Unexpectedly, S1P was not a chemoattractant for patrolling monocytes and had no significant effect on their viability in vitro. Moreover, the disruption of S1P gradients in vivo did not alter Ly6C $\mathrm{C}^{-}$monocyte trafficking and viability. These data suggest that S1PR5 regulates the trafficking of monocytes via a mechanism independent of S1P gradients.

Keywords: Bone marrow - Patrolling monocytes - Sphingosine-1 phosphate - Survival • Trafficking

\section{Introduction}

Blood monocytes are bone marrow (BM) derived phagocytic cells that play an important role in innate immunity against different classes of pathogens [1]. Human and mouse monocytes have been subdivided into at least two subsets on the basis of expression of
CD14 and CD16 (human) and Ly6C (mouse) and several functional, migratory [2] and transcriptomic [3-5] parameters.

Mouse $\mathrm{Ly} 6 \mathrm{C}^{+}$monocytes are classical inflammatory monocytes, equivalent to human $\mathrm{CD} 14^{+} \mathrm{CD} 16^{-}$monocytes, as recently confirmed by gene profiling experiments $[4,5]$. They are rapidly recruited to inflamed tissues in response to CC chemokine 
Receptor 2 (CCR2) [6] or CCR6 [7] ligands. During infection by various pathogens (intracellular bacteria, parasites, or viruses), they differentiate into TNF/iNOS producing dendritic cells (Tip-DCs) that produce large amounts of TNF- $\alpha$, reactive oxygen species, and nitric oxide [8]. They can also differentiate into other types of DCs in other inflammatory contexts [9]. Moreover, Ly6C $^{+}$ monocytes are involved in atherosclerosis and can also differentiate into macrophages or myeloid suppressor cells [2].

The role of $\mathrm{Ly} 6 \mathrm{C}^{-}$monocytes remains more elusive. Ly6C $\mathrm{C}^{-}$ monocytes express high levels of $\mathrm{CX}_{3} \mathrm{CR} 1$, which allows them to patrol healthy tissues through long-range crawling on the surface of blood endothelium at the luminal side [10], in response to membrane-anchored endothelial $\mathrm{CX}_{3}$ CL1 [11]. This interaction is also required for their survival [11]. They express low levels of CCR2 and migrate less efficiently to inflamed tissues than inflammatory monocytes [12]. They have been proposed to be precursors of resident macrophage populations [13]. Moreover, their human equivalent, the $\mathrm{CD} 16^{+} \mathrm{CD} 14^{\mathrm{dim}}$ monocytes respond to virus infection through TLR7 and TLR8 (where TLR is Toll-like receptor) and produce TNF- $\alpha$, IL-1 $\beta$, and CC chemokine Ligand 3 (CCL3) [4]. A recent article also reported that $\mathrm{Ly}_{6} \mathrm{C}^{-}$monocytes were uniquely equipped with high levels of Fc $\gamma$ receptors involved in antibodydependent cell cytotoxicity such as Fc $\gamma \mathrm{R} 1$ and Fc $\gamma \mathrm{R} 4$ [14]. Finally, they could also have a role in tissue repair and angiogenesis [13].

Monocytes are produced in the BM from macrophage-DC precursor [13]. Upon development, monocytes reach the blood circulation via BM sinusoids. Egress of $\mathrm{Ly} 6 \mathrm{C}^{+}$monocytes from BM has been shown to be dependent on CCR2. This egress is weak under steady-state conditions but increases massively upon inflammation induced by bacterial infection [6]. During infections, low concentrations of TLR ligands in the bloodstream drive CCR2-dependent emigration of monocytes from the BM. BM mesenchymal stem cells and CXC chemokine ligand 12 abundant reticular cells rapidly express CCL2 in response to TLR ligands or bacterial infection and induce monocyte egress into the blood [15]. How Ly6C $\mathrm{C}^{-}$monocytes reach the peripheral blood is however still unknown.

Here, we report that Ly6 $\mathrm{C}^{-}$monocytes expressed high levels of sphingosine-1 phosphate receptor 5 (S1PR5), previously involved in BM egress of natural killer (NK) cells [16]. S1pr5 $5^{-/-}$mice lack peripheral Ly6 $6 \mathrm{C}^{-}$monocytes. Our data support a role for S1PR5 together with CCR2 in their egress from the BM. Modulation of extracellular S1P levels did not affect monocyte trafficking to the blood while it reduced T-cell egress from lymphoid organs, showing that S1P receptors regulate the trafficking of monocytes and lymphocytes using different mechanisms.

\section{Results}

\section{S1PR5 is required for the presence of Ly6C- monocytes in the periphery}

We measured using quantitative RT-PCR the expression of all S1PR in different lymphocyte and monocyte populations sorted by flow cytometry from the BM. S1PR5 showed the highest expres-

\section{$\square$ Ly6C $^{+} \square$ Ly6C- $\square$ T cells $\square$ NK cells}

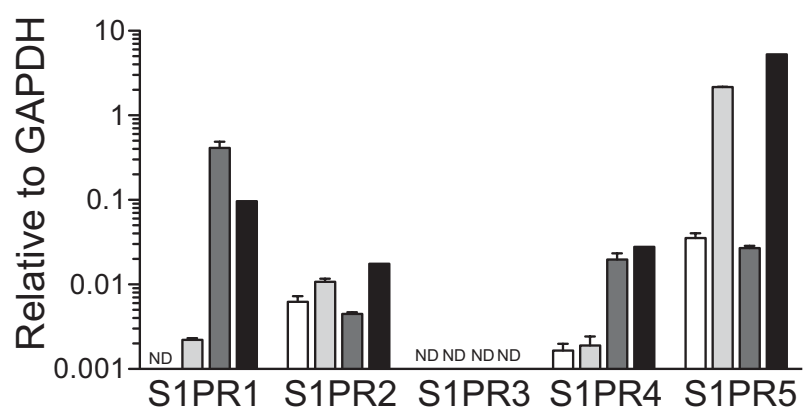

Figure 1. Expression of S1P receptors in different immune cell types. Ly6C $\mathrm{C}^{+}$and Ly6C- monocytes, NK cells, and T cells were sorted by flow cytometry from the BM and their mRNA expression of S1P receptors was measured using semi quantitative RT-PCR, relative to GAPDH. Results are representative of two independent FACS sorting experiments and show the mean + SD of triplicate PCRs for each S1P receptor.

sion in monocyte subsets. S1PR5 was expressed 30 times higher in Ly6C $\mathrm{C}^{-}$monocytes than in Ly6C $\mathrm{C}^{+}$monocytes (Fig. 1). A similar difference in S1PR5 expression between monocyte subsets has been measured using microarrays by the Immgen consortium (http://www.immgen.org/databrowser/index.html) [17]. S1PR5 levels in $\mathrm{Ly}^{-} \mathrm{C}^{-}$monocytes were comparable to those in NK cells (Fig. 1), in which S1PR5 plays a role in BM egress [16].

To investigate the function of S1PR5 in monocytes, we first compared the percentage of monocyte subsets in the blood of wild-type (WT) and S1pr5 $5^{-/-}$mice [18] by flow cytometry. Results in Figure 2A-C showed a significant reduction of $\mathrm{Ly}^{-} \mathrm{C}^{-}$ monocytes in the blood of $S 1 \mathrm{pr}^{-/-}$mice. This reduction was observed both in $S 1 p r 5^{-/-}$female (Fig. 2A and B) and male mice (Fig. 2C). S1pr5 $5^{+/-}$heterozygous mice also showed a mild phenotype (Fig. 2B). A strong reduction in the frequency of $\mathrm{Ly}^{-} \mathrm{C}^{-}$ monocytes was also observed in the spleen, which is known to be an important reservoir for this subset [19] (Fig. 2D), in the lymph nodes and in non-lymphoid organs such as the lung, liver, and kidney (Fig. 2E). By contrast, the percentage of Ly6C $\mathrm{C}^{-}$monocytes appeared normal in the BM of $S 1 p r 5^{-/-}$mice (Fig. 2F). Moreover, the percentage of $\mathrm{Ly}_{6} \mathrm{C}^{+}$monocytes was normal in all lymphoid organs of $S 1 p r 5^{-/-}$mice tested (Fig. 2, all panels).

To test if the role of S1PR5 in monocytes was cell-intrinsic, we generated mixed BM chimeras by reconstituting lethally irradiated mice with equal amounts of BM from WT $\left(\mathrm{CD} 45.1^{+}\right)$and S1pr5 ${ }^{-/-}$ $\left(C D 45.2^{+}\right)$mice. Six weeks after reconstitution, we measured CD45.1 and CD45.2 expression in different immune subsets in the blood and BM, and calculated the corresponding $S 1 \mathrm{pr} 5^{-/-}$to WT ratio for each subset. As previously reported [20], for mature $\mathrm{NK}$ (mNK) cells, this ratio was very high in the BM and very low in the blood (Fig. 3, left panel), reflecting the important role of S1PR5 in NK cell exit from the BM. For Ly6C ${ }^{+}$monocytes, the $S 1 \mathrm{pr}^{-/-}$to WT ratio was nearly 1 in both blood and BM (Fig. 3, right panel), confirming the absence of a role of S1PR5 in this 
A

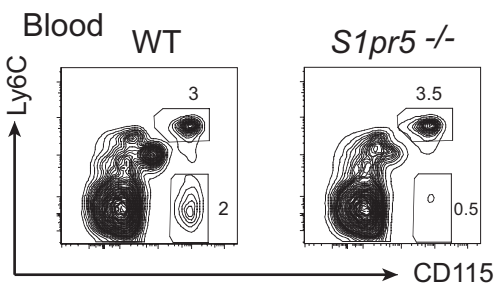

B Blood, females

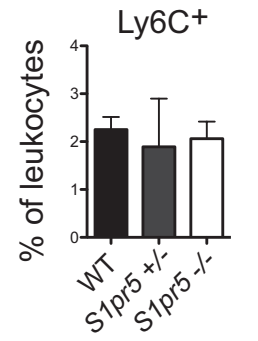

C Blood, males

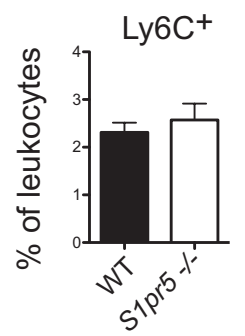

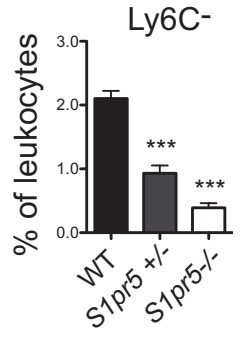

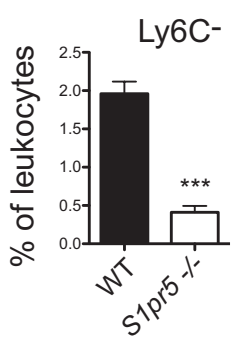

D Spleen
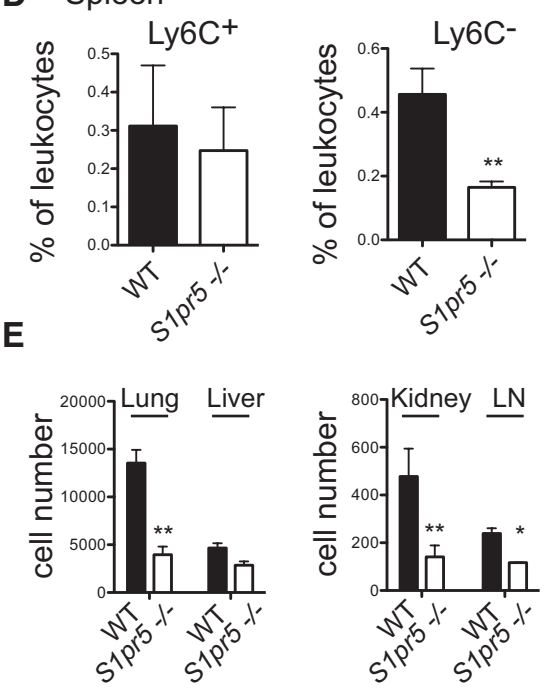

F Bone marrow

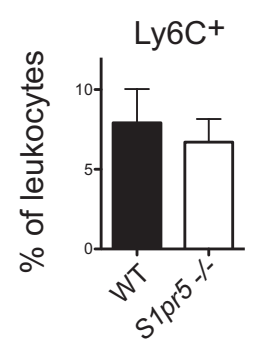

Figure 2. S1pr5 $5^{-/-}$mice lack peripheral Ly6C $\mathrm{C}^{-}$monocytes. (A-F) Flow cytometric analyses of the percentage or absolute number of Ly6C $\mathrm{C}^{+}$and Ly6C $\mathrm{C}^{-}$monocytes in (A-C) the blood, (D) the spleen, (E) lung, liver, kidney, lymph nodes, and (F) the BM of WT and S1pr5 $5^{-1-}$ mice, as indicated. (A) A representative flow cytometric analysis in the blood of WT and S1pr5 $5^{-/-}$mice is shown. (C-F) Data are shown as the mean + SD percentage or absolute number of monocyte subsets among total leukocytes in groups of 5 (spleen) to 20 (blood and BM) age- and sex-matched mice, and are pooled from three experiments performed. ${ }^{*} p<0.05,{ }^{* *} p<0.01,{ }^{* * *} p<0.001$, t-test.

subset. By contrast, for Ly6C $\mathrm{C}^{-}$monocytes, the $S 1 \mathrm{pr}^{-/-}$to WT ratio was near 0.5 in the BM and 0.1 in the blood (Fig. 3, left panel). These data suggest that S1PR5 is important both for the development of $\mathrm{Ly} 6 \mathrm{C}^{-}$monocytes and for their trafficking or their survival at the periphery.

\section{S1PR5 and CCR2 but not $\mathrm{CX}_{3} \mathrm{CR} 1$ are essential for localization of $\mathrm{Ly}_{6 \mathrm{C}^{-}}$monocytes to BM sinusoids}

The paucity of patrolling monocytes in the periphery of $S 1 \mathrm{pr} 5^{-/-}$ mice could be explained by a role of this receptor either in their egress from the BM or in their survival at the periphery. To try and discriminate between both hypotheses, we performed a series of experiments using $\mathrm{Cx}_{3} \mathrm{Cr} 1^{g \mathrm{fp} / \mathrm{gfp}}$ and $\mathrm{Ccr} 2^{-/-}$mice as controls. Indeed, $\mathrm{CX}_{3} \mathrm{CR} 1$ has been shown to regulate peripheral survival of patrolling monocytes but is devoid of chemotactic activity involved in BM egress. Reciprocally, CCR2 is essential for monocyte egress from the BM but is not involved in their survival. The distribution of Ly6C $\mathrm{C}^{-}$monocytes in $C x_{3} c r 1^{g f p / g f p}$ and $C c r 2^{-/-}$mice is in fact very similar to that of $S 1 \mathrm{pr}^{-/-}$mice, with a near normal frequency
BM Blood

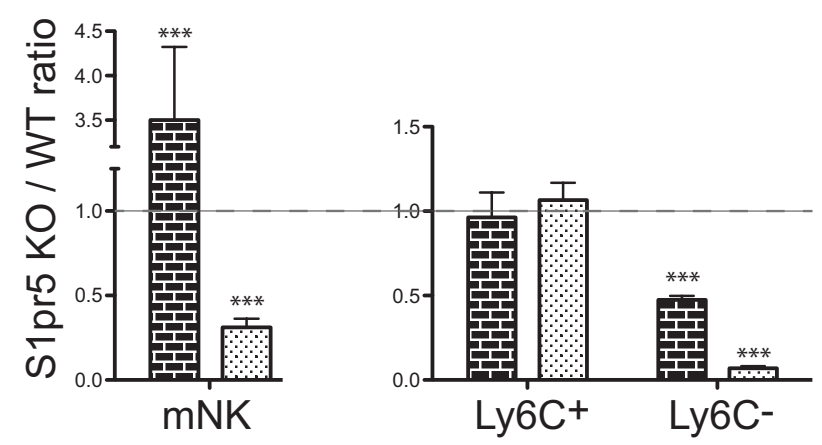

Figure 3. The role of S1PR5 in Ly6C- monocytes is cell-intrinsic. Mixed $\mathrm{BM}$ chimeric mice were generated by reconstituting irradiated CD45.1 $\times$ CD45.2 mice with a 1:1 mixture of WT CD45.1 BM cells and S1pr5 $5^{-1-}$ CD45.2 BM cells as indicated. Eight weeks after reconstitution, the percentage of CD45.2 positive cells in the indicated subsets was measured by flow cytometry in the blood and BM. Results are shown as the mean $+\mathrm{SD}$ of four mice in each group and are expressed as the ratio between S1 $\mathrm{pr}^{-/-}$and WT cells for each subset, as indicated. This experiment is representative of four independent experiments performed. ${ }^{* * *} p<0.001$, t-test. 
A

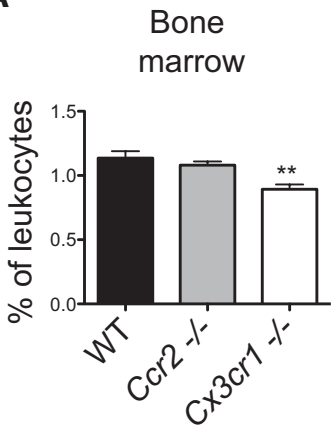

Bone

C

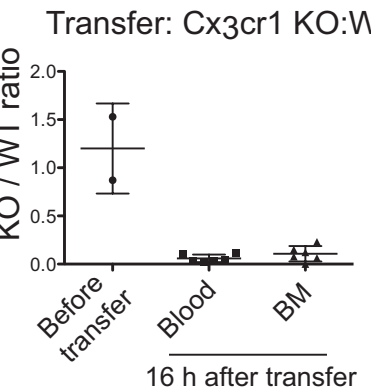

\section{E}

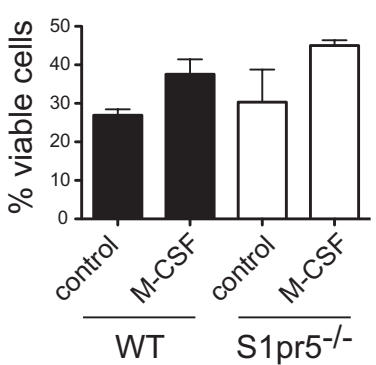

Blood

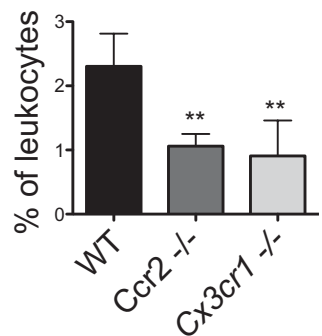

B

\section{BM sinusoids}

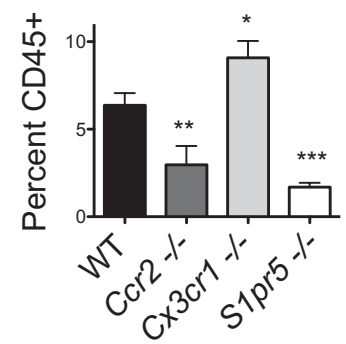

D

F

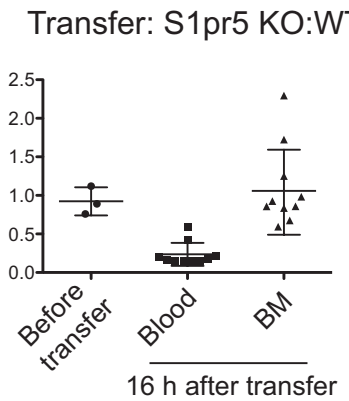

CX3cr1-
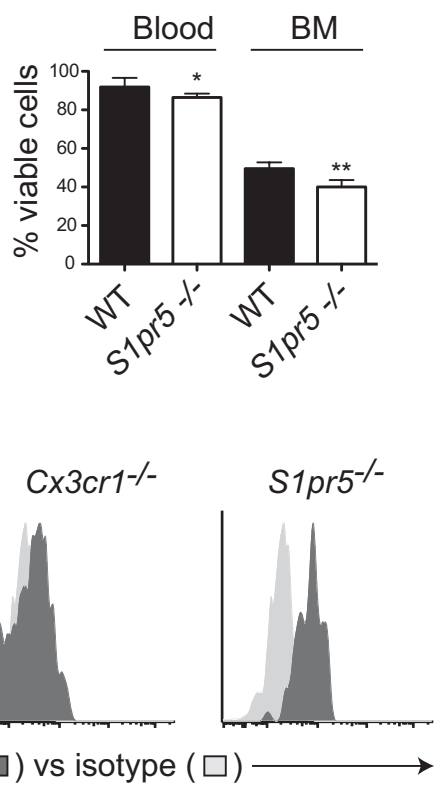

Figure 4. S1PR5 promotes BM egress of Ly6C $\mathrm{C}^{-}$monocytes and not survival. (A) Flow cytometric analyses of the percentage of Ly6 $\mathrm{C}^{-}$monocytes in the blood and the BM of WT, $\mathrm{Cx}_{3} \mathrm{Cr} 1 \mathrm{gfp} / \mathrm{gfp}$ and $\mathrm{Ccr} 2^{-/-}$mice. Data are shown as the mean + SD of $4-10$ mice in each group. (B) In some experiments, the frequency of sinusoidal cells $\left(\mathrm{CD} 45^{+}\right)$within total BM Ly6C ${ }^{-}$monocytes was measured. Data are shown as mean $+\mathrm{SD}$ of five mice in each group. (A, B) Data shown are pooled from three experiments performed. (C) Adoptive transfer of BM cells from C $x_{3} \mathrm{Cr} 19 f \mathrm{fp} / \mathrm{gfp}$ mice and CD45.1 WT mice or S1pr5 ${ }^{-1-}$ and CD45.1 WT mice (1:1 ratio) into CD45.1 $\times$ CD45.2 recipient mice. Sixteen hours after transfer the number of Ly6C ${ }^{-}$monocytes from each donor was measured in the BM and blood of recipient mice by flow cytometry. Results are expressed as a ratio between KO and CD45.1 WT Ly6C- monocytes, for each combination, before and after transfer in the BM and blood. Each symbol represents an individual recipient mouse. Data are pooled from three independent experiments. (D) Freshly isolated blood and BM cells from WT S1pr5 ${ }^{-1-}$ chimeric mice were stained with Annexin V and 7-AAD and antibodies to detect monocyte cells. The frequency of viable Ly6C ${ }^{-}$monocytes was determined by flow cytometry. Data

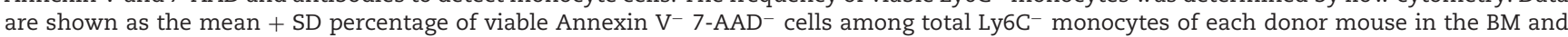
blood. Results are representative of three independent experiments with at least three mice in each group. (E) Ly6 $\mathrm{C}^{-}$monocytes were sorted from $\mathrm{BM}$ cell suspensions by flow cytometry and cultured for $24 \mathrm{~h}$ in medium alone or supplemented with M-CSF as indicated. Data are shown as the mean \pm SD viability of cells as assessed by Annexin V / 7-AAD staining and are pooled from three independent experiments. (F) Flow cytometric measurement of intracellular Bcl2 expression in blood Ly6C $\mathrm{C}^{-}$monocytes from different mouse strains as indicated. Data are representative of $n=3$ mice in each group. ${ }^{*} p<0.05,{ }^{* *} p<0.01,{ }^{* * *} p<0.001$, t-test.

in the $\mathrm{BM}$ and a low frequency of these cells at the periphery (Fig. 4A).

First, we compared the frequency of sinusoidal Ly6C $\mathrm{C}^{-}$monocytes in the BM of WT, $\mathrm{Cx}_{3} \mathrm{Cr} 1^{g f p / g f p}, \mathrm{Ccr} 2^{-/-}$, and $S 1 p r 5^{-/-}$ mice using in vivo labeling of sinusoidal cells. This measurement has been shown to be proportional to the BM exit rate. Indeed, newly developed BM leukocytes transit from the BM parenchyma through the endothelium and into the BM sinusoids where they are transiently retained until their release into the blood circulation. Results presented in Fig. 4B showed that the percentage of sinusoidal Ly6 $\mathrm{C}^{-}$monocytes was significantly decreased in the $\mathrm{BM}$ of $S 1 \mathrm{pr} 5^{-/-}$or $C \mathrm{cr} 2^{-/-}$mice compared to the BM of WT mice. 
By contrast, the percentage of sinusoidal Ly6C $\mathrm{C}^{-}$monocytes was significantly increased in the BM of $C x_{3} C r 1^{g f p / g f p}$ mice compared to the BM of WT mice. These results support a role for S1PR5 in the migration of Ly6 $\mathrm{C}^{-}$monocytes from the parenchyma to the sinusoidal compartment of the BM, a process essential for exit from the BM. This process could be negatively regulated by $\mathrm{CX}_{3} \mathrm{CR} 1$, perhaps as a result of adhesive properties of $\mathrm{CX}_{3} \mathrm{CR} 1$.

Second, we compared the fate of monocytes of different genotypes adoptively transferred into recipient mice. We performed intravenous injection of a 1:1 mixture of WT (CD45.1) and $S 1 p r 5^{-/-}$or $C x_{3} c r 1^{g f p / g f p}$ (CD45.2) BM cells into recipient WT (CD45.1 × CD45.2) mice. Sixteen hours after transfer, we measured the frequency of donor monocyte subsets in the blood and the BM of recipient mice. We calculated the ratio between WT and $\mathrm{KO}$ donors for each subset before transfer and $16 \mathrm{~h}$ after transfer in the blood and the BM. C $x_{3} c r 1^{g f p / g f p} \mathrm{Ly} 6 \mathrm{C}^{-}$monocytes were barely detectable in both $\mathrm{BM}$ and blood of recipient mice, confirming the important role of $\mathrm{CX}_{3} \mathrm{CR} 1$ in the survival of $\mathrm{Ly}_{6} \mathrm{C}^{-}$ monocytes (Fig. 4C, left panel). By contrast, transferred $S 1 \mathrm{pr}^{-/-}$ Ly6C $\mathrm{C}^{-}$monocytes were almost absent from the blood but were represented at similar frequency as $\mathrm{WT} \mathrm{Ly}^{-} \mathrm{C}^{-}$monocytes in the $\mathrm{BM}$ of recipient mice (Fig. 4C, right panel). These data support a role for S1PR5 in the egress of Ly6C $\mathrm{C}^{-}$monocytes rather than in their survival.

Third, we compared the ex vivo viability of WT and $S 1 p r 5^{-/-}$ Ly6C $\mathrm{C}^{-}$monocytes in the blood and BM of WT $S 1 p r 5^{-/-}$chimeric mice using AnnexinV/7-AAD staining. In both compartments, the viability of $S 1 p r 5^{-/-}$Ly6C $\mathrm{C}^{-}$monocytes was slightly lower than that of WT Ly6C ${ }^{-}$monocytes (Fig. 4D). Moreover, irrespective of the mouse genotype, the viability of $\mathrm{Ly}^{6} \mathrm{C}^{-}$monocytes was lower in the BM than in the blood. We also assessed viability of WT and S1pr5 $5^{-1-}$ Ly6C $^{-}$monocytes sorted by flow cytometry and cultured in the presence or absence of M-CSF. After $24 \mathrm{~h}$, the viability of WT and S1pr5 $5^{-/-}$Ly6C $\mathrm{C}^{-}$monocytes was similar in both culture conditions (Fig. 4E). Finally, we measured the expression of $\mathrm{Bcl} 2$, an important anti-apoptotic molecule that has been shown to be down regulated in $C x_{3} c r 1^{g f p / g f p}$ Ly $6 \mathrm{C}^{-}$monocytes and to regulate their survival. The expression of $\mathrm{Bcl} 2$ was similar in Ly6C $\mathrm{C}^{-}$monocytes from WT and $S 1 p r 5^{-/-}$mice but it was reduced in $C x_{3} c r 1^{g f p / g f p}{\text { Ly } 6 C^{-}}$monocytes (Fig. $4 \mathrm{~F}$ ), as previously reported [21].

Altogether, this series of in vivo experiments demonstrate that $\mathrm{CX}_{3} \mathrm{CR} 1$ and S1PR5 regulate homeostasis of the peripheral pool of Ly6C $\mathrm{C}^{-}$monocytes via different mechanisms. While $\mathrm{CX}_{3} \mathrm{CR} 1$ is clearly involved in their survival, S1PR5 is rather implicated in their egress from the BM although it may also contribute indirectly in their survival.

\section{Modulation of S1P levels does not alter the trafficking and survival of Ly6C- monocytes}

Finally, we investigated the role of S1P in the physiology of Ly6C monocytes using in vitro and in vivo experiments.
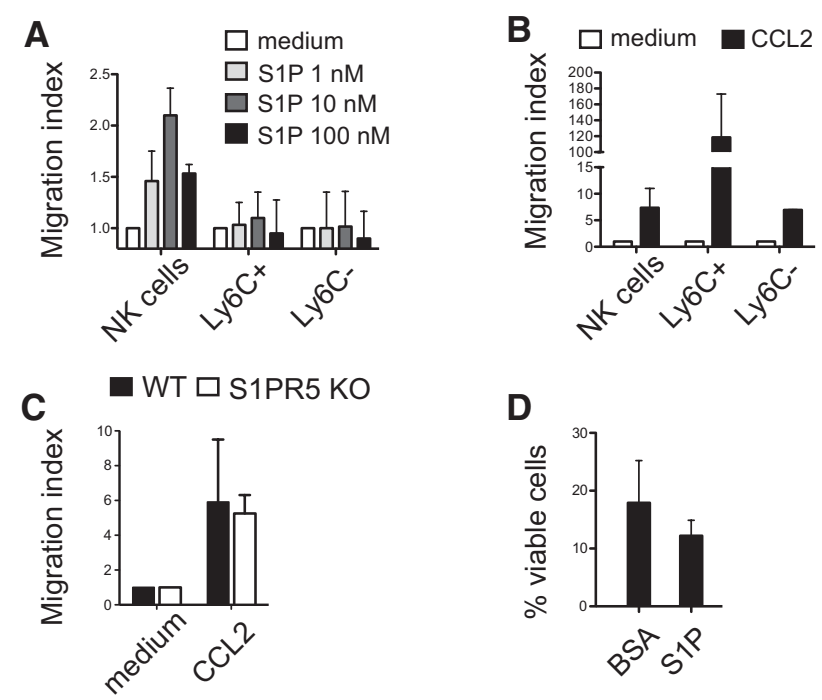

E

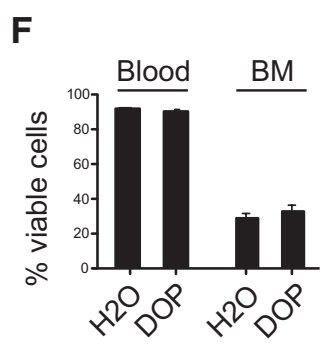

Figure 5. S1P gradient is not required for homeostasis of Ly6 $\mathrm{C}^{-}$monocytes. (A and B) Transwell migration assay of purified Ly6C ${ }^{+}$and Ly6C ${ }^{-}$ monocytes or NK cells (A) in response to different concentrations of S1P or (B) in response to CCL2 $(50 \mathrm{ng} / \mathrm{mL})$ as indicated. Values are reported as the mean ratio $+\mathrm{SD}$ between the number of cells that migrated during the $2 \mathrm{~h}$ incubation with S1P or CCL2 and the number of cells migrating in the control (no chemokine) condition. Results are representative of three independent experiments. (C) Transwell migration of BM Ly6C ${ }^{-}$ monocytes from WT and S1pr5 $5^{-/-}$mice in response to CCL2 gradients, as indicated. (D) Ly6C $\mathrm{C}^{-}$monocytes were sorted from BM cell suspensions by flow cytometry and cultured for $24 \mathrm{~h}$ in medium without FCS supplemented with BSA, in the presence or absence of S1P, as indicated. Data are shown as mean + SD of six samples pooled from three independent experiments. ( $E$ and $F$ ) $C 57 \mathrm{BL} / 6$ mice were treated with DOP given in the drinking water for 3 or 10 days. (E) The percentage of $\mathrm{Ly} 6 \mathrm{C}^{+}$and Ly6C $\mathrm{C}^{-}$monocytes and the percentage of $\mathrm{CD}^{+} \mathrm{T}$ cells was measured on days 3 (top) and 10 (bottom) by flow cytometry. (F) The viability of Ly6C ${ }^{-}$-monocytes was measured after 3 days of treatment in the BM and in the blood. Results are shown as the mean + SD of six mice in each group and are pooled from three experiments performed. ${ }^{* * *} p<0.001$, t-test.

In vitro, we measured responsiveness of monocytes to S1P gradients in chemotaxis chambers. No consistent migration of either population of monocytes was observed (Fig. 5A), whereas both monocyte populations migrated in response to CCL2 gradients (Fig. 5B). In the same experiments, NK cells migrated in response 
to both S1P and CCL2 gradients (Fig. 5A and B), as previously reported [16]. WT and $S 1 p r 5^{-/-} \mathrm{Ly}_{6 \mathrm{C}^{-}}$monocytes migrated equally to CCL2 gradients, excluding a possible cross talk between CCR2 and S1PR5 (Fig. 5C). We also cultured Ly6C ${ }^{-}$monocytes with S1P at concentrations similar to those observed in vivo. The addition of S1P at any concentration did not change monocyte viability in vitro (Fig. 5D and data not shown).

Next, we treated mice with the sphingosine lyase inhibitor deoxypyridoxine (DOP), which has been shown to dramatically increase S1P levels in tissues and disrupt S1P gradients in vivo [22]. Upon treatment with DOP, peripheral T-cell numbers dropped, as previously reported [22]. However, DOP had no effect on the trafficking or the number of Ly6 $\mathrm{C}^{-}$monocytes (Fig. 5E) and NK cells [22] even after prolonged (10 days) treatment (Fig. 5E). The ex vivo viability of blood and BM Ly6C ${ }^{-}$monocytes was not modified either (Fig. 5F).

Altogether, these results suggest that S1P and S1P gradients are not involved in monocyte survival and unexpectedly not in their trafficking.

\section{Discussion}

In this article, we report for the first time a high expression of S1PR5 in patrolling monocytes and the paucity of these cells in the peripheral compartment of $S 1 p r 5^{-/-}$mice. The following body of evidences supports a role for S1PR5 in BM egress of patrolling monocytes: (i) We previously showed that S1PR5 was involved in NK-cell egress from the BM to blood [20,23]. Moreover, several other members of the family of S1P receptors (S1PR1, S1PR3) are clearly involved in egress of different leukocyte subsets from central and peripheral lymphoid organs [24]. (ii) Ly6C $\mathrm{C}^{-}$monocytes are reduced in BM sinusoids of $S 1 p r 5^{-/-}$mice, whereas they are preserved, or even slightly increased in $C x_{3} c r 1^{g f p / g f p}$ mice, which only exhibit impaired survival of Ly6C ${ }^{-}$monocytes at the periphery. (iii) The phenotype of $S 1 p r 5^{-/-}$mice is very similar to that of $\mathrm{Ccr} 2^{-/-}$mice in which monocyte egress from the BM has been shown to be clearly impaired [15]. In particular, the number of $\mathrm{Ly}_{6 \mathrm{C}} \mathrm{C}^{-}$monocytes was normal in the BM of $S 1 \mathrm{pr} 5^{-/-}$and $\mathrm{Ccr} 2^{-/-}$ mice but reduced in the blood circulation and in BM sinusoids. (iv) Upon adoptive transfer into recipient mice, $S 1 p r 5^{-/-}$Ly6C $^{-}$ monocytes recirculate to the $\mathrm{BM}$ and rapidly disappear from the blood circulation. For comparison, $C x_{3} \operatorname{cr} 1^{f f p / g f p}$ Ly6C- monocytes do not survive either in the BM or in the blood after transfer.

An intriguing observation is the absence of accumulation of $S 1 p r 5^{-/-}$Ly6C ${ }^{-}$monocytes in the BM of $S 1 p r 5^{-/-}$mice or WT $S 1 \mathrm{pr}^{-/-} \mathrm{BM}$ chimeric mice. A similar phenomenon (i.e. lack of accumulation of $\mathrm{Ly}_{6 \mathrm{C}} \mathrm{C}^{-}$monocytes) was also observed in $\mathrm{Ccr} 2^{-/-}$ mice and WT $C c r 2^{-/-}$BM chimeric mice. This suggests that the trafficking machinery of Ly6C $\mathrm{C}^{-}$monocytes regulates somehow the developmental fitness of these cells and that an impairment of this machinery results in an impaired survival. As a matter of fact, we found that the ex vivo viability of $\mathrm{Ly} \mathrm{C}^{-}$monocytes in the $\mathrm{BM}$ was very low, confirming previous findings [25]. It is therefore possible that an impairment of their trafficking by means of CCR2 or S1PR5 deletion could further decrease the viability of these fragile cells.

In vivo modulation of S1P levels by pharmacological means did not alter homeostasis of Ly6 $\mathrm{C}^{-}$monocytes (this report), while they dramatically reduced the number of $\mathrm{T}$ cells in circulation. These results show that S1P receptors operate through different modes of action in monocytes and in T cells. Several hypotheses could explain this paradox. First, the role of S1PR5 in Ly6C ${ }^{-}$monocytes could be S1P-independent. Other physiological ligands for this receptor have not yet been described but specific S1PR5 analogs binding with high affinity to this receptor have been synthesized [26], and may therefore exist in vivo. Second, it has been reported that S1PR5 could act as a constitutively active receptor [27] like other G-protein-coupled receptors [28]. S1PR5 was in fact shown to decrease adenylyl cyclase and ERK activity in several cell lines in the absence of S1P, inducing cell rounding and detachment without promoting apoptosis [27]. This effect could contribute or even induce cell migration by preventing strong attachment to the stromal substrate of the BM. In this scenario, S1PR5 would not be a chemotactic receptor in monocytes, which would explain why we could not detect migration of these cells in response to S1P gradients in vitro. An alternative possibility could be that the form of S1P physiologically active in monocytes is different from the one we use in vitro. In fact, S1P can be found under different forms in vivo that could have differential activities on leukocyte subsets. Further studies are required to test these points. It remains also to be determined whether S1PR5 acts differently in monocytes and NK cells. Indeed, S1pr5 $5^{-/-}$mice lack both peripheral NK cells and $\mathrm{Ly}^{-} \mathrm{C}^{-}$monocytes but only NK cells accumulate in the $\mathrm{BM}$ of these mice and migrate in vitro in response to S1P.

Altogether, our findings shed light on the long-sought mechanisms of exit of $\mathrm{Ly}^{-} \mathrm{C}^{-}$monocytes from the BM $[12,29]$. They also show fundamental differences between both monocyte subsets, as the exit of $\mathrm{Ly} \mathrm{C}^{+}$monocytes from the BM relies only on pro-inflammatory signals mediated by CCR2 [6] whereas that of Ly6C $\mathrm{C}^{-}$monocytes is primarily mediated by S1PR5. Furthermore, $S 1 \mathrm{pr}^{-/-}$mice constitute an interesting model to study the role of Ly6C $\mathrm{C}^{-}$monocytes in immunity, a point that remains unclear.

\section{Materials and methods}

\section{Mice and reagents}

WT C57BL/ 6 mice were purchased from Charles River Laboratories (L'Arbresle, France). S1pr5 $5^{-/-}$mice [18], Ccr $2^{-/-}$[30], and $C x_{3} c r 1^{g f p / g f p}$ mice [31] have been previously described. In some experiments, we also used C57BL/6 CD45.1 mice or C57BL/6 CD45.1 $\times$ CD45.2 mice that were bred in our animal house. Female mice 8-24 week-old were used unless specified. DOP (Sigma, St. Louis, MO, USA) was provided in the drinking water (30 $\mu \mathrm{g} / \mathrm{mL}$ ) supplemented with glucose. Experimental procedures 
and mice housing were approved by the local Ethics Committee and carried out according to the French and European laws.

\section{Generation of BM chimera}

C57BL/ 6 CD45.1 $\times$ CD45.2 mice were irradiated twice at $450 \mathrm{rad}$ within a 4-h interval. Four hours after the last irradiation, they received an intravenous injection of a 1:1 mixture of BM cells from WT CD45.1 and S1 pr5 ${ }^{-/-}$CD45.2 mice. BM chimeras were analyzed 6-12 weeks after reconstitution.

\section{In vivo labeling of sinusoidal lymphocytes}

This technique was previously described [32]. Briefly, mice were injected intravenously with $1 \mu \mathrm{g}$ anti-CD45 Mab (30F11) coupled to phycoerythrin (PE) or PE-cyanin-5 (BD Biosciences, San Jose, USA). Mice were sacrificed 2 min after antibody injection. BM was then collected and analyzed by flow cytometry.

\section{Adoptive transfers}

BM cells from WT CD45.1 and S1pr5 ${ }^{-/-}$or $C x_{3} c r 1^{g f p / g f p}(\mathrm{CD} 45.2)$ mice were prepared and mixed at a 1:1 ratio before intravenous injection $\left(1 \times 10^{7}\right.$ cells of each genotype in PBS) into anesthetized CD45.1 $\times$ CD45.2 C57BL/6 mice. Sixteen hours later, mice were sacrificed, blood and bone marrow was collected and the percentage of monocyte subsets of each donor mice was measured by flow cytometry after staining for CD45.1 and CD45.2 expression.

\section{Cell viability}

Cell viability was measured in ex vivo isolated cell suspensions using Annexin V and 7-AAD staining (BD Biosciences) and flow cytometry.

\section{Antibodies and flow cytometry}

BM, spleen, lung, lymph node, kidney, and blood cells were isolated and stained as previously described [33]. Cell counts were determined using an accuri C6 flow cytometer (BD Accuri Cytometers, Ann Arbor, MI, USA). Monocytes were identified as CD115 ${ }^{+}$

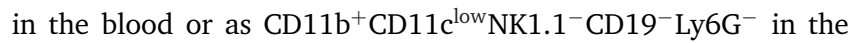
$\mathrm{BM}$ and spleen. The following Mabs from eBioscience (San Diego, CA, USA) or BD Biosciences (Becton Dickinson, San Jose, USA) were used: anti-CD115 (AFS98), anti-Ly6C (HK1.4), anti-Ly6G (1A8), anti-CD19 (ebio1D3), anti-CD3 (145-2C11), anti-NK1.1 (PK136), anti NKp46 (29A1.4), anti-CD11b (M1/70), anti-CD45.1 (A20), anti CD45.2 (104), and relevant isotype controls. Bcl2 expression was measured using a commercial kit (BD Biosciences) according to the manufacturer's instructions. Flow cytometry was carried out on a FACS Canto, a FACS Canto II or a FACS LSR II (Becton Dickinson).

\section{Chemotaxis assays}

For S1P migration assays, monocytes were purified from BM cells using a negative selection procedure. Briefly, freshly isolated BM cells were stained with purified antibodies against CD4, CD8, CD19, and Ly6G (eBioscience). Cells were then incubated with magnetic beads covered with goat anti-rat IgG (Qiagen, Hilden, Germany) before magnetic separation. After washing steps, cells were then suspended in RPMI1640 supplemented with $4 \mathrm{mg} / \mathrm{mL}$ fatty acid-free bovine albumin (Sigma). The same medium was used to prepare S1P (Sigma) at $10^{-8} \mathrm{M}$ or CCL2 at $50 \mathrm{ng} / \mathrm{mL}$ (R\&D systems). Cell migration was measured in Transwell chambers (Costar, Cambridge, MA, USA) with $5-\mu \mathrm{m}$ pore-width polycarbonate filters. After $2 \mathrm{~h}$, transmigrated cells were stained for CD3, NK1.1, CD27 and CD11b or CD11b, Ly6G and Ly6C and counted by flow cytometry as described previously [16].

\section{Quantitative RT-PCR}

Lymphocyte or monocyte subsets stained with the appropriate antibodies were sorted using a FACS Aria cell sorter (Becton Dickinson). RNA was extracted with the RNeasy micro kit (Qiagen), which includes treatment with DNase I. We used Superscript II reverse transcriptase (Invitrogen, Carlsbad, CA, USA) to generate cDNA for RT-PCR. PCR was carried out with a SybrGreenbased kit (FastStart Universal SYBR Green Master, Roche, Basel, Switzerland) on a StepOne plus instrument (Applied biosystems, Carlsbad, CA, USA). Primers were designed using the oligoperfect software (Invitrogen, Carlsbad, CA, USA). The following primers were used: S1pr1 (F: AAATGCCCCAACGGAGACTCTG, R: TTGCTGCGGCTAAATTCCATGC), S1pr2 (F: CCCAACTCCGGGACATAGA, R: ACAGCCAGTGGTTGGTTTTG), S1pr3 (F: TCAGTGGTTCATCATGCTGG, R: CAGGTCTTCCTTGACCTTCG), S1pr4 (F: AAGACCAGCCGTGTGTATGG, R: TCAGCACGGTGTTGAGTAGC), S1pr5 (F: GCCTGGTGCCTACTGCTACAG, R: CCTCCGTCGCTGGCTATTTCC), Gapdh (F: GCATGGCCTTCCGTGTTC, R: TGTCATCATACTTGGCAGGTTTCT). S1PR expression level in the different cell subsets was normalized to GAPDH expression levels.

\section{In vitro culture of Ly6C- monocytes}

Ly6 $\mathrm{C}^{-}$monocytes were sorted by flow cytometry using a FACS Aria cell sorter (Becton Dickinson). They were cultured in flat bottom 96 well plates (25000/condition) in duplicates. For cultures with M-CSF, the culture medium was supplemented with 10\% FCS in the presence or absence of $5 \%$ of an M-CSF-containing cell culture supernatant. In some experiments, cells were resuspended in medium supplemented with $4 \mathrm{mg} / \mathrm{mL}$ fatty acid-free bovine albumin (Sigma). The same medium was used to prepare S1P 
(Sigma), which was added or not to the cultures at a concentration of $10^{-6} \mathrm{M}$.

\section{Statistical analyses}

Statistical analyses were performed using two-tailed $t$-tests or nonparametric tests when appropriate. These tests were run on the Prism software (GraphPad, La Jolla, CA, USA). Levels of significance are expressed as $p$-values $\left(* p<0.05,{ }^{* *} p<0.01\right.$, $* * * p<0.001)$.

Acknowledgments: Authors thank the Plateau de Biologie Expérimentale de la Souris, and the flow cytometry facility of the SFR Biosciences Gerland. We also thank Andrew Calver (GlaxoSmithKline) for providing S1PR5 KO mice and Steffen Jung for the $\mathrm{CX}_{3} \mathrm{CR} 1^{\text {gfp/gfp }}$ mice. The T. W. lab is supported by the FINOVI foundation, Agence Nationale de la Recherche (ANR JC sphinks), European Research council (ERC-Stg 281025), Institut National de la Santé et de la Recherche Médicale (INSERM).

\section{References}

1 Serbina, N. V., Jia, T., Hohl, T. M. and Pamer, E. G., Monocyte-mediated defense against microbial pathogens. Annu. Rev. Immunol. 2008. 26: 421-452.

2 Auffray, C., Sieweke, M. H. and Geissmann, F., Blood monocytes: development, heterogeneity, and relationship with dendritic cells. Annu. Rev. Immunol. 2009. 27: 669-692.

3 Ancuta, P., Liu, K.-Y., Misra, V., Wacleche, V. S., Gosselin, A., Zhou, X. and Gabuzda, D., Transcriptional profiling reveals developmental relationship and distinct biological functions of CD16 + and CD16- monocyte subsets. BMC Genomics. 2009. 10: 403-422.

4 Cros, J., Cagnard, N., Woollard, K., Patey, N., Zhang, S.-Y., Senechal, B., Puel, A. et al., Human CD14dim monocytes patrol and sense nucleic acids and viruses via TLR7 and TLR8 receptors. Immunity 2010. 33: 375-386.

5 Ingersoll, M. A., Spanbroek, R., Lottaz, C., Gautier, E. L., Frankenberger, M., Hoffmann, R., Lang, R. et al., Comparison of gene expression profiles between human and mouse monocyte subsets. Blood 2010. 115: e10-e19.

6 Serbina, N. V. and Pamer, E. G., Monocyte emigration from bone marrow during bacterial infection requires signals mediated by chemokine receptor CCR2. Nat. Immunol. 2006. 7: 311-317.

7 Le Borgne, M., Etchart, N., Goubier, A., Lira, S. A., Sirard, J. C., van Rooijen, N., Caux, C. et al., Dendritic cells rapidly recruited into epithelial tissues via CCR6/CCL20 are responsible for CD8+ T cell crosspriming in vivo. Immunity 2006. 24: 191-201.
8 Serbina, N. V., Salazar-Mather, T. P., Biron, C. A., Kuziel, W. A. and Pamer, E. G., TNF/iNOS-producing dendritic cells mediate innate immune defense against bacterial infection. Immunity 2003. 19: 59-70.

9 Tacke, F. and Randolph, G. J., Migratory fate and differentiation of blood monocyte subsets. Immunobiology. 2006. 211: 609-618.

10 Auffray, C., Fogg, D., Garfa, M., Elain, G., Join-Lambert, O., Kayal, S., Sarnacki, S. et al., Monitoring of blood vessels and tissues by a population of monocytes with patrolling behavior. Science 2007. 317: 666-670.

11 Kim, K.-W., Vallon-Eberhard, A., Zigmond, E., Farache, J., Shezen, E., Shakhar, G., Ludwig, A. et al., In vivo structure/function and expression analysis of the CX3C chemokine fractalkine. Blood 2011. 118: e156-e167.

12 Shi, C. and Pamer, E. G., Monocyte recruitment during infection and inflammation. Nat. Rev. Immunol. 2011. 11: 762-774.

13 Geissmann, F., Manz, M. G., Jung, S., Sieweke, M. H., Merad, M. and Ley, $\mathrm{K}$., Development of monocytes, macrophages, and dendritic cells. Science 2010. 327: 656-661.

14 Biburger, M., Aschermann, S., Schwab, I., Lux, A., Albert, H., Danzer, H., Woigk, M. et al., Monocyte subsets responsible for immunoglobulin G-dependent effector functions in vivo. Immunity 2011. 35: 932-944.

15 Shi, C., Jia, T., Mendez-Ferrer, S., Hohl, T. M., Serbina, N. V., Lipuma, L., Leiner, I. et al., Bone marrow mesenchymal stem and progenitor cells induce monocyte emigration in response to circulating toll-like receptor ligands. Immunity 2011. 34: 590-601.

16 Mayol, K., Biajoux, V., Marvel, J., Balabanian, K. and Walzer, T., Sequential desensitization of CXCR4 and S1P5 controls natural killer cell trafficking. Blood 2011. 118: 4863-4871.

17 Heng, T. S. P., Painter, M. W. and Immunological Genome Project Consortium, The Immunological Genome Project: networks of gene expression in immune cells. Nat. Immunol. 2008. 9: 1091-1094.

18 Jaillard, C., Harrison, S., Stankoff, B., Aigrot, M. S., Calver, A. R., Duddy, G., Walsh, F. S. et al., Edg8/S1P5: an oligodendroglial receptor with dual function on process retraction and cell survival. J. Neurosci. 2005. 25: 1459-1469.

19 Swirski, F. K., Nahrendorf, M., Etzrodt, M., Wildgruber, M., CortezRetamozo, V., Panizzi, P., Figueiredo, J.-L. et al., Identification of splenic reservoir monocytes and their deployment to inflammatory sites. Science 2009. 325: 612-626.

20 Walzer, T., Chiossone, L., Chaix, J., Calver, A., Carozzo, C., GarrigueAntar, L., Jacques, Y. B. et al., Natural killer cell trafficking in vivo requires a dedicated sphingosine 1-phosphate receptor. Nat. Immunol. 2007. 8: 1337-1344.

21 Jakubzick, C., Tacke, F., Ginhoux, F., Wagers, A. J., van Rooijen, N., Mack, M., Merad, M. and Randolph, G. J., Blood monocyte subsets differentially give rise to CD103+ and CD103- pulmonary dendritic cell populations. J. Immunol. 2008. 180: 3019-3027.

22 Schwab, S. R., Pereira, J. P., Matloubian, M., Xu, Y., Huang, Y. and Cyster, J. G., Lymphocyte sequestration through S1P lyase inhibition and disruption of S1P gradients. Science 2005. 309: 1735-1739.

23 Jenne, C. N., Enders, A., Rivera, R., Watson, S. R., Bankovich, A. J., Pereira, J. P., Xu, Y. et al., T-bet-dependent S1P5 expression in NK cells promotes egress from lymph nodes and bone marrow. J. Exp. Med. 2009. 206: 2469-2481.

24 Cyster, J. G. and Schwab, S. R., Sphingosine-1-Phosphate and lymphocyte egress from lymphoid organs. Annu. Rev. Immunol. 2012. 30: 69-94.

25 Hanna, R. N., Carlin, L. M., Hubbeling, H. G., Nackiewicz, D., Green, A. M., Punt, J. A., Geissmann, F. et al., The transcription factor NR4A1 (Nur77) controls bone marrow differentiation and the survival of Ly6C- monocytes. Nat. Immunol. 2011. 12: 778-785. 
26 Mattes, H., Dev, K. K., Bouhelal, R., Barske, C., Gasparini, F., Guerini, D., Mir, A. K. et al., Design and synthesis of selective and potent orally active S1P5 agonists. Chem. Med. Chem. 2010. 5: 1693-1696.

27 Niedernberg, A., Blaukat, A., Schöneberg, T. and Kostenis, E., Regulated and constitutive activation of specific signalling pathways by the human S1P5 receptor. Br. J. Pharmacol. 2003. 138: 481-493.

28 Rosenbaum, D. M., Rasmussen, S. G. F. and Kobilka, B. K., The structure and function of G-protein-coupled receptors. Nature 2009. 459: 356-363.

29 Ingersoll, M. A., Platt, A. M., Potteaux, S. and Randolph, G. J., Monocyte trafficking in acute and chronic inflammation. Trends Immunol. 2011. 32: 470-477.

30 Boring, L., Gosling, J., Chensue, S. W., Kunkel, S. L., Farese, Jr, R. V., Broxmeyer, H. E. and Charo, I. F., Impaired monocyte migration and reduced type 1 (Th1) cytokine responses in C-C chemokine receptor 2 knockout mice. J. Clin. Invest. 1997. 100: 2552-2561.

31 Jung, S., Aliberti, J., Graemmel, P., Sunshine, M. J., Kreutzberg, G. W., Sher, A. and Littman, D. R., Analysis of fractalkine receptor CX(3)CR1 function by targeted deletion and green fluorescent protein reporter gene insertion. Mol. Cell Biol. 2000. 20: 4106-4114.
32 Pereira, J. P., An, J., Xu, Y., Huang, Y. and Cyster, J. G., Cannabinoid receptor 2 mediates the retention of immature B cells in bone marrow sinusoids. Nat. Immunol. 2009. 10: 403-411.

33 Walzer, T., Bléry, M., Chaix, J., Fuseri, N., Chasson, L., Robbins, S. H., Jaeger, S. et al., Identification, activation, and selective in vivo ablation of mouse NK cells via NKp46. Proc. Natl. Acad. Sci. U S A. 2007. 104: 3384-3389.

Abbreviations: DOP: deoxypyridoxine - S1P: sphingosine-1 phosphate . S1PR5: sphingosine-1 phosphate receptor 5

Full correspondence: Dr. Thierry Walzer, INSERM U1111, 21 avenue Tony Garnier, Lyon, France 69007

Fax: +33-(0) 437282341

e-mail: thierry.walzer@inserm.fr 